This item was submitted to Loughborough's Research Repository by the author.

Items in Figshare are protected by copyright, with all rights reserved, unless otherwise indicated.

\title{
Measuring the efficacy of positioning aids for capturing 3D data in different clothing configurations and postures with a high-resolution whole-body scanner
}

\section{PLEASE CITE THE PUBLISHED VERSION}

https://doi.org/10.1016/j.measurement.2020.108519

\section{PUBLISHER}

Elsevier

\section{VERSION}

AM (Accepted Manuscript)

\section{PUBLISHER STATEMENT}

This paper was accepted for publication in the journal Measurement and the definitive published version is available at https://doi.org/10.1016/j.measurement.2020.108519

\section{LICENCE}

CC BY-NC-ND 4.0

\section{REPOSITORY RECORD}

Schwarz-Muller, Frank, Russell Marshall, Steve Summerskill, and Christoph Poredda. 2020. "Measuring the Efficacy of Positioning Aids for Capturing 3D Data in Different Clothing Configurations and Postures with a High-resolution Whole-body Scanner". Loughborough University. https://hdl.handle.net/2134/13020482.v1. 


\title{
Measuring the efficacy of positioning aids for capturing 3D data in different clothing configurations and postures with a high-resolution whole-body scanner
}

\section{Author names and affiliations}

Dr Frank Schwarz-Müller ${ }^{\text {ab*, }}$

Dr Russell Marshall a,

Dr Steve Summerskill a,

Christoph Poredda ${ }^{\mathrm{c}}$,
FrankSchwarzMueller@,bundeswehr.org

$\underline{\text { R.Marshall@,1boro.ac.uk }}$

S.J.Summerskill2@,1boro.ac.uk

ChristophPoredda@bundeswehr.org

a School of Design and Creative Arts, Loughborough University, Leicestershire LE11 3TU, UK

${ }^{b}$ Federal Office of Bundeswehr Equipment, Information Technology and In-Service Support (BAAINBw), Ferdinand Sauerbruch Straße 1, Kolonnenweg, 56073 Koblenz, Germany

${ }^{c}$ Bundeswehr Technical Centre for Land-Based Vehicle Systems, Engineer and General Field Equipment (WTD 41), Kolonnenweg, 54296 Trier, Germany

\begin{abstract}
Numerous surveys have been conducted worldwide to capture 3D anthropometric data of individuals scanned in tight underwear. However, such semi-nude data are inadequate for designing workspaces for specialised user populations who wear protective clothing and equipment. Determining the offset between semi-nude and clothed configurations requires the same individual to be repeatedly scanned in exactly the same posture. Specifically, for the use in a high-resolution 3D body scanner, positioning aids for the standing and seated posture were developed to stabilise the posture during the scanning process without compromising data integrity. The mean absolute variability $(M A V)$ index was introduced to determine the efficacy of the positioning aids. It was shown that the positioning aids efficiently reduce the variability in fore-and-aft and side-to-side directions. This way the precondition was created for the precise superimposition of scans permitting the offset between diverse clothing configurations to be determined.
\end{abstract}

\section{Key words}

Body scanning, Positioning aid, Clothed anthropometry, Human variability

\footnotetext{
* Corresponding author, Federal Office of Bundeswehr Equipment, Information Technology and In-Service Support (BAAINBw), Ferdinand Sauerbruch Straße 1, 56073 Koblenz, Germany, F.SchwarzMuller@lboro.ac.uk
} 


\section{Introduction}

\subsection{Challenges of clothed anthropometry}

State-of-the-art 3D whole-body scanners allow high resolution scans to be captured and more than 100 body dimensions to be extracted without physical contact in less than 10 seconds [1]. The digital 3D image of an individual generated by the scanning system can be revisited for analysis and research at any time [2]. These are valuable benefits over manual measurements utilising traditional instruments such as callipers, stadiometers and anthropometers, which have resulted in the use of 3D body scanning technology in numerous anthropometric surveys worldwide [3-5]. All these surveys have in common that semi-nude anthropometric data were collected, i.e. from individuals wearing tight-fitting underwear. Therefore, there is a good understanding of the seminude anthropometry of a wide range of different user populations. However, the semi-nude body dimensions are inadequate for designing workstations in vehicles, ships and airplanes for specialised user populations who wear bulky and multi-layered protective clothing that results in increased body size [6,7]. This applies to user populations such as firefighters, search and rescue units, police, soldiers, military aircrew, astronauts etc. Conducting large-scale surveys to collect $3 \mathrm{D}$ clothed anthropometric data in the same way as semi-nude data is hardly viable in terms of cost, time and human resources. A large number of different encumbered user populations, the almost infinite number of possible clothing configurations and the short replacement cycles for protective equipment call for a more expedient and efficient approach. Ascertaining clothed anthropometric data in addition to semi-nude data of a limited representative sample of a user population allows the relationships between the two configurations to be determined. Adding this offset, between standardised semi-nude dimensions and clothed dimensions, to the existing semi-nude database permits statistically valid estimates of space requirements for workstations to be established [8].

\subsection{Determining the offset between semi-nude and clothed data}

As early as 1986 Kamal [9] attempted to determine the offset between semi-nude and clothed anthropometric dimensions in the field of student health care. Since it sometimes proved to be impractical to perform anthropometric measurements semi-nude, measurements were taken on clothed students. Using tools such as a measuring tape and a micrometre screw gauge, he established the mathematical relation between circumferential semi-nude and clothed dimensions of individuals wearing standardized clothing of known thickness. The introduction of the 3D scanning technology has revolutionised anthropometry as it allows body shapes to be captured in seconds without physical contact. Psikuta et al. [10] used 3D data obtained with two laser body scanning systems to determine the airgap in clothing. To this end they superimposed the scans taken of a rigid mannequin with and without clothing. The 3D comparison between the two scans resulted in a $3 \mathrm{D}$ colour map quantifying the offset between the nude and clothed condition of the mannequin 
highly accurately. Utilising this methodology the data were generated, which Mert et al. [11] used to validate the garment simulation software Fashionizer (MIRAlab, University of Geneva, Switzerland). The software was then employed to determine the offset with and without clothing for an avatar while walking. This way, the contact areas of the clothing on the body were identified and the air gap was quantified at the points where the clothing is not in direct contact with the body. Latest research efforts focus on the use of 3D clothed body scans or sequences of such scans while the individual is moving (4D) for estimating the nude body shape. In their study, Park et al. [12] estimated the nude body shape underneath clothing using a statistical body shape model (SBSM). The SBSM was fitted to 3D scans taken from soldiers in four different clothing configurations. Employing an inscribed-fitting method, the nude 3D body shape was determined. Yang et al. [13] and Zhang et al. [14] presented methodologies to estimate the nude body shape underneath loose clothing using a sequence of 3D scans of an individual in motion and fitting a space or a parametric body model to it. Irrespective of whether 3D or 4D methods are applied, with the estimates of the nude $3 \mathrm{D}$ body shape available, it is then possible to estimate the offset between nude and clothed configurations.

For the determination of the offset between semi-nude and clothed populations, all presented methods have their limitations. While the use of a rigid mannequin allows the offset to be determined accurately, the shape and size variations occurring in user populations cannot be addressed adequately. Conversely, the estimates of the nude body shape are based on 3D or 4D scans of individuals and therefore consider human shape and size variations. However, they are model-based estimates and not actual measurements, which is why deviations from the true body shape have to be accepted.

In theory, it would also be possible to determine the offset utilising technologies that can penetrate the clothing, such as magnetic resonance imaging (MRI), computed tomography (CT), X-ray or millimetre waves. However, high investment costs, limited accessibility and, above all, ethical aspects preclude their use for this purpose.

Against this background, it seems to be favourable to use surface scanners to determine the offset between semi-nude and clothed configurations by scanning the same person in both configurations and measuring the differences in space claim applying differential techniques to the superimposed scans.

\subsection{Precise replication of posture by mans of a positioning aid}

A prerequisite for establishing the differences between semi-nude and clothed data is the precise replication of postures when an individual is repeatedly scanned in different clothing configurations (Figure 1). However, when scanning an individual standing freely on a scanner platform, human variability, i.e. postural variations and body sway prevents the offset from being determined with 
the required accuracy. In research, different approaches have been taken to overcome this deficiency. Ballester et al. [15] and Colaianni et al. [16] presented software-supported approaches

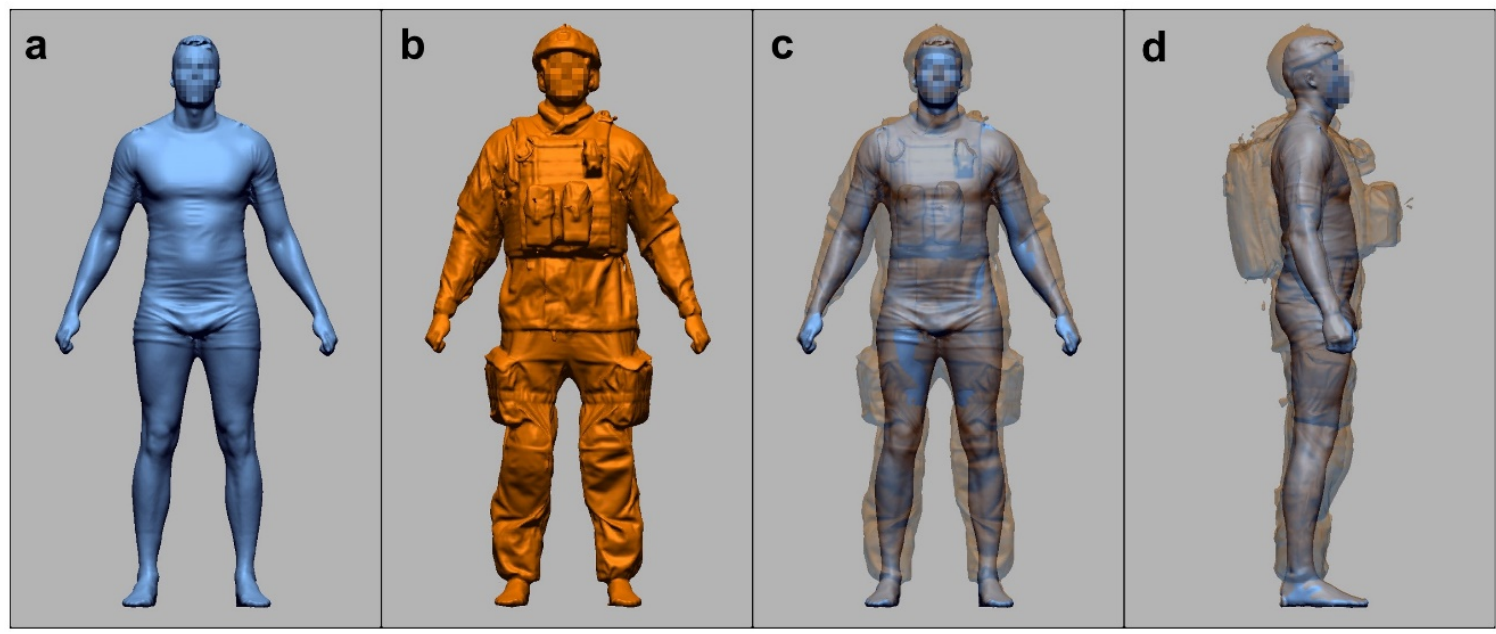

Figure 1: Superimposition of scans (c, d) to determine the difference between clothing configurations (a, b)

for harmonising the posture of semi-nude scan data, making use of animation principles. A distinct step of their software-supported harmonisation of postures is template fitting based on anatomical landmarks. In addition, the transfer of the template skeleton to the target mesh requires landmarks or very similar shapes and surfaces. When wearing protective clothing and equipment, many of the landmarks are covered and differences in shapes and surfaces between different clothing configurations (e.g. semi-nude and fully clothed) are significant. Thus software-supported posture harmonisation is not an option in the field of clothed anthropometry.

In contrast to software-supported posture harmonisation, mechanical positioning aids potentially reduce postural variability of both semi-nude and clothed participants. In earlier research only a few attempts have been made to counter human variability, i.e. body sway and postural variability by introducing rudimentary mechanical positioning aids $[17,18]$. A reason for a lack of research in this field might have been the fact that mechanical positioning aids can compromise the scan data by obscuring relevant body parts and therefore impede the use of software for the automatic extraction of body dimensions from the scans. More recently, Schwarz-Müller et al. [19] showed that a positioning aid predominantly made of Plexiglas, allows the human variability to be reduced to less than $10 \mathrm{~mm}$ when scanned repeatedly with a 3D laser body scanner. In addition, the design principles applied prevent the positioning aid from being visible on the scan and thus impairing the automatic extraction of body measurements.

\subsection{Measuring the efficacy of positioning aids}

Modern 3D body scanning systems include software packages that automatically extract body dimensions from a scan. Researchers have taken advantage of this feature in a number of studies to compare the precision of scan derived measurements of different 3D body scanners $[20,21]$ or to compare the precision of scan derived measurements against data ascertained using 
traditional methods [22-26]. Typically, three repeated scans of a participant are taken, and a defined set of linear and circumferential body dimensions are extracted from each scan. Then the mean absolute difference $(M A D)$ between the repeated scan derived measurements for each body dimension is calculated as an index for the precision of the measurement. In earlier research the authors [19] used the $M A D$ index to determine the efficacy of a positioning aid by comparing the precision of the measurements with and without positioning aid. Utilising the $M A D$ index to analyse the differences between repeated scan derived measurements has the advantage that no further tools but the scanning hardware and software are needed for the analysis. However, the $M A D$ does not provide directional information, indicating to what extent the variability manifests in fore-and-aft (sagittal axis) and side-to-side directions (transverse axis). This is considered to be essential for the evaluation of a positioning aid and the development of recommendations to further optimise its design. Furthermore, the landmark detection software of the scanners is error-prone [27], which can have a decisive impact on the precision of the scan derived measurements. To overcome these deficiencies a more direct measurement method was introduced to determine the efficacy of positioning aids. To this end seven horizontal sections were taken through three superimposed scans of the same individual wearing tight underwear. The body locations for the seven sections were chosen to allow the human variability of the trunk as well as the extremities, i.e. head, arms and legs, to be measured. The maximum distance between the contours of the three sections in sagittal and transverse directions at each body location served as measure to compare the human variability with and without positioning aid. In this way, a methodology for determining the effectiveness of positioning aids was established, which is less error-prone than the $M A D$ index due to the shorter measurement chain and at the same time provides directional information.

\subsection{Contribution of this paper}

A latest-generation 3D laser body scanning system (VITUS ${ }^{\text {bodyscan }}$, VITRONIC, Wiesbaden, Germany) was acquired specifically for a study to develop methods for evaluating the offset between different clothing configurations. As explained in 1.3, the harmonisation of postures during the scanning process is a prerequisite for the accurate measurement of the offset. In previous research, a positioning aid was developed to demonstrate that the posture of repeatedly scanned semi-nude individuals can be reliably reproduced [19]. This positioning aid was further developed for this study to accommodate not only semi-nude individuals, but also individuals wearing personal protective clothing and equipment (PPE). The design process included a fundamental redesign of the fixation elements to ensure that, despite the high resolution of the new scanner, they remain invisible on scans and are robust enough for military personnel with their PPE. This included but was not limited to mounting the fixation elements as well as their adjustment 
mechanism to a frame outside the scan volume, redesigning the fixation elements for arms, hip and feet, introducing a fixation for the shoulder and optimising their positioning relative to the body to avoid artefacts on the scan.. In addition, a new positioning aid for the seated posture was to be developed to exploit the new scanner's ability to scan people while seated.

This article presents results and findings of $\mathrm{PhD}$ research conducted by Loughborough University in the professional setting of the PhD student at a Technical Test Centre of the German Armed Forces.

The paper

- describes the design of the newly developed positioning aids for the use in a high-resolution scanner in both the standing and the seated posture,

- presents the result of a study to determine the efficacy of the two positioning aids by measuring the mean absolute variability $(M A V)$ manifesting in the sagittal and transverse axes,

- proposes establishing the $M A V$ as measure of efficacy for positioning aids in reducing postural variability, thereby quantifying their influence on the accuracy of measurement chains.

This way the basis for evaluating the offset between semi-nude and clothed configurations was created, which was subject of an anthropometric study the results of which will be presented in a separate paper.

\section{Methods}

\subsection{Development of positioning aids for a high-resolution scanner}

\subsubsection{Positioning aid for the standing posture}

In previous work, the authors described the development of a mechanical positioning aid for the standing posture $[19,28]$. This positioning aid incorporated fixation elements for head, arms and feet predominately made of Plexiglas and a taught horizontal steel wire constituting a hip fixation. Installed in a VITUS ${ }^{\text {smart }}$ LC laser scanner (VITRONIC, Wiesbaden, Germany) and a NX16 structured light scanner ( $\mathrm{TC}^{2}$, Cary, USA) these fixation elements effectively reduced postural variability, were not visible on scans and did not compromise the automatic extraction of body dimensions from scans. The research also indicated that the variability of shoulders and arms could be further reduced by incorporating a supporting element, to avoid participants twisting their shoulders.

Since this initial research body scanning technology has improved, resulting in high resolution scanners. In this study the VITUS ${ }^{\text {bodyscan }}$ scanning system (VITRONIC, Wiesbaden, Germany) was 
used, which constitutes a major technological leap compared to its predecessor as it generates a three-dimensional colour image of the scanned object with a 43-times higher point density. Initial tests with the positioning aid as presented in the above article showed that the fixation elements made of taught steel wire and parts of the Plexiglas elements became visible on the scans. Therefore, the positioning aid had to undergo a design-iteration to make it suitable for the use in the new scanner. The following set of functional requirements was established for the development of the positioning aid for the standing posture:

- Fixation elements to be provided for head, shoulder, arms, hip and feet

- Fixation elements to be made of translucent Plexiglas tubes to reduce the reflection of the light emitted by the scanner

- Alternatively, fixation elements to be made of wire or cord with a diameter below the resolution of the scanner or non-reflective material

- Design and adjustment elements of the fixtures to be relocated as far as practicable outside the scanning volume

- Refraction artefacts of the fixation elements on the scanned body as well as artefacts on the scans to be minimised through the optimised placement of the fixation elements

- The adjustment range of the fixation elements should be as large as the scan volume allows, but must include the $5^{\text {th }}$ percentile German female and the $95^{\text {th }}$ percentile German male ranging in stature from $157.1 \mathrm{~cm}$ to $191,0 \mathrm{~cm}$ as a minimum requirement (informed by the results of the survey "sizeGERMANY" conducted 2007/2008)

Guided by these functional requirements, the fixation elements of the positioning aid have been optimised and led to the positioning aid shown in Figure 2 (left). A sample scan of the subjects restrained by the positioning shows that its elements remain invisible.

The design process resulted in the following design solutions for the fixtures of head, shoulders, arms, hip and feet, which are either fixed to a self-supported frame made of aluminium profiles located outside the scanning volume or the scanner platform (Table 1).

Head fixture -A mouthpiece incorporating a stop collar was fixed to one end of a solid Plexiglas rod. The opposite end of the rod was inserted in holes drilled through the bottom end of a vertical Plexiglas tube (also referred to as centre tube). To avoid inconsistencies in head tilt, it is important that the experimenter adjusts the height of the mouthpiece such that the head is in the Frankfort plane.

Shoulder fixture -To avoid the participants twisting their shoulder during the scanning process, a shoulder fixation in form of a horizontal Plexiglas tube is located over the shoulder on either side. While one end of the tube is fixed to the frame the other end is equipped with a pivotal clip allowing 
the connection of the shoulder fixation to the clothing of the participant. When the shoulder is twisted, force feedback is created, which is supposed to cause the individual to turn back the shoulders until it has disappeared.

Arm fixture - The arm fixation is formed by a horizontal Plexiglas support beam holding the frontfacing handle at one end. The other end is slid over an aluminium tube with a base plate, which is mounted to the aluminium frame.

Hip fixture -Four parallel cords with a diameter of $1 \mathrm{~mm}$ were used as hip fixture. The cords of exactly the same length are attached at both ends to a square aluminium tube. These are fastened with tensioning screws to a base plate, which is mounted to the aluminium profiles of the frame.

Foot fixtures - Heel cups cut off from commercially available foot gauges and fitted with two guide pins constituted the foot fixtures. Holes drilled into the scanner platform to accommodate the guide pins allowed the foot fixtures to be adjusted in the sagittal directions. In transverse direction the holes were drilled in a distance ensuring the feet of the participants to be positioned shoulder width apart as stipulated by the scanner manufacturer.
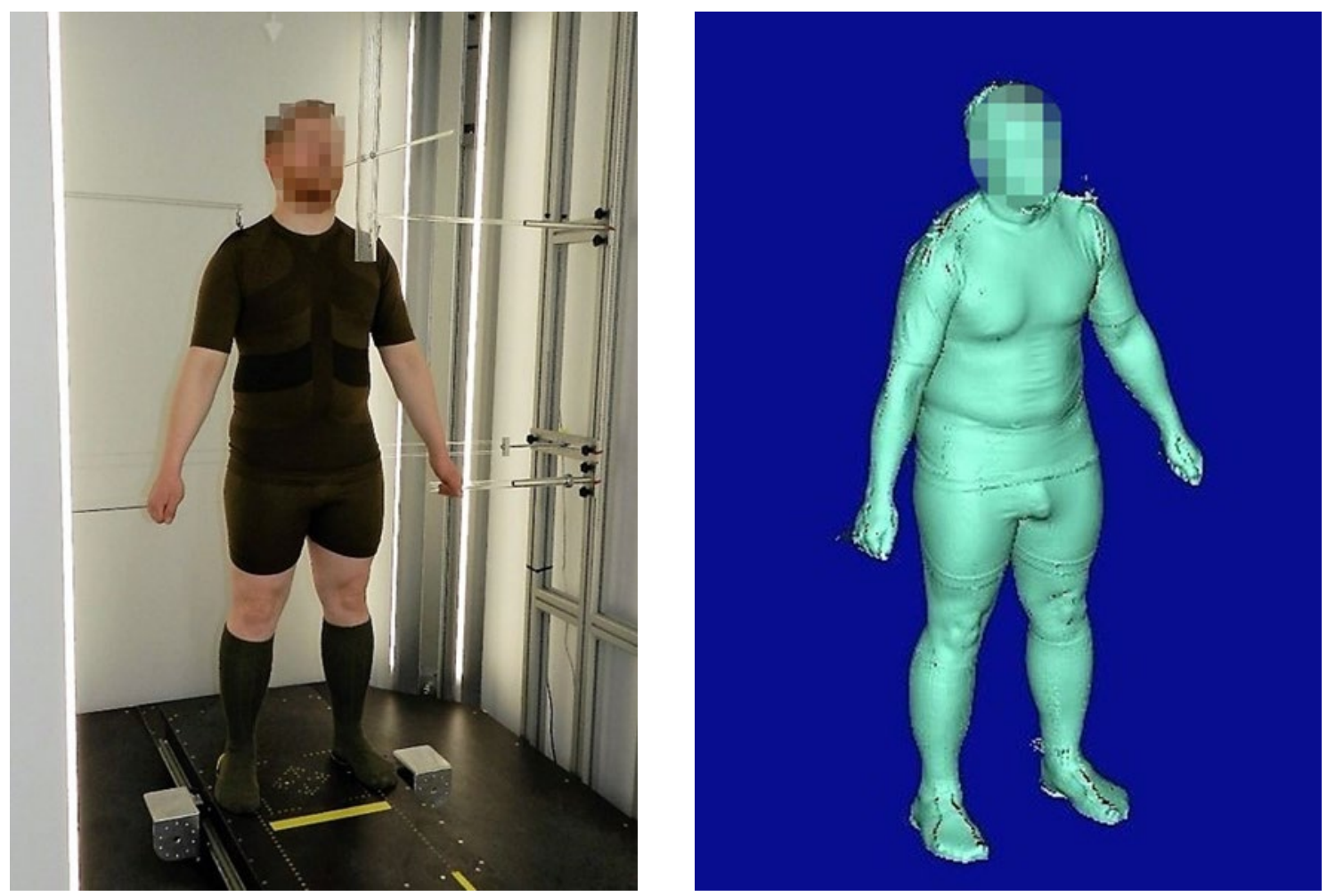

Figure 2: Positioning aid for the standing posture (left); Sample scan (right)

To find out whether the redesign efforts led to the desired result, the above design solutions had to undergo a visibility test. Table 1 gives an overview of the test results. 
Table 1: Evaluation of the fixation elements of the positioning aid for the standing posture in terms of visibility

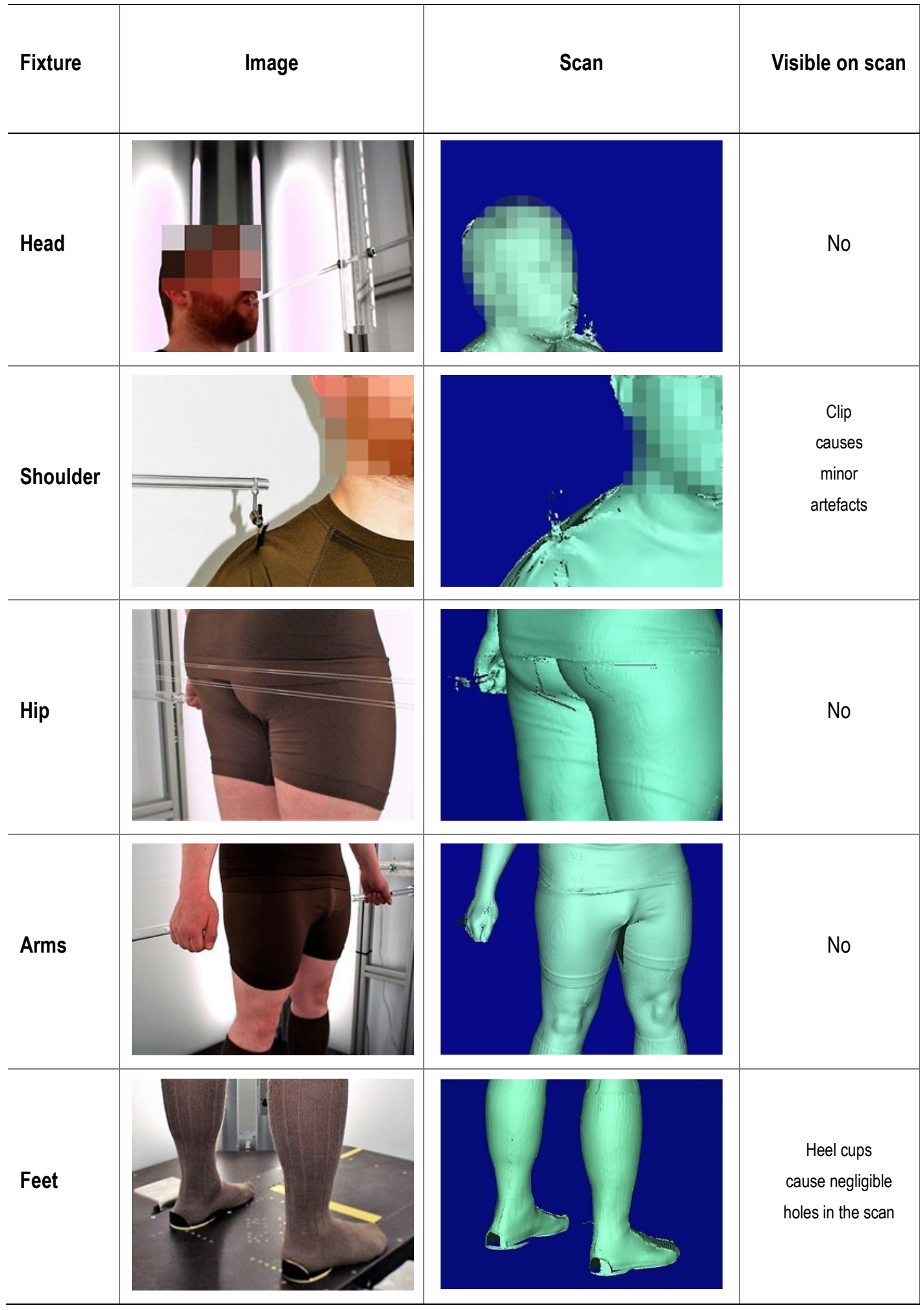


The adjustment ranges of the fixation elements relative to the scanner platform. are depicted in Figure 3.
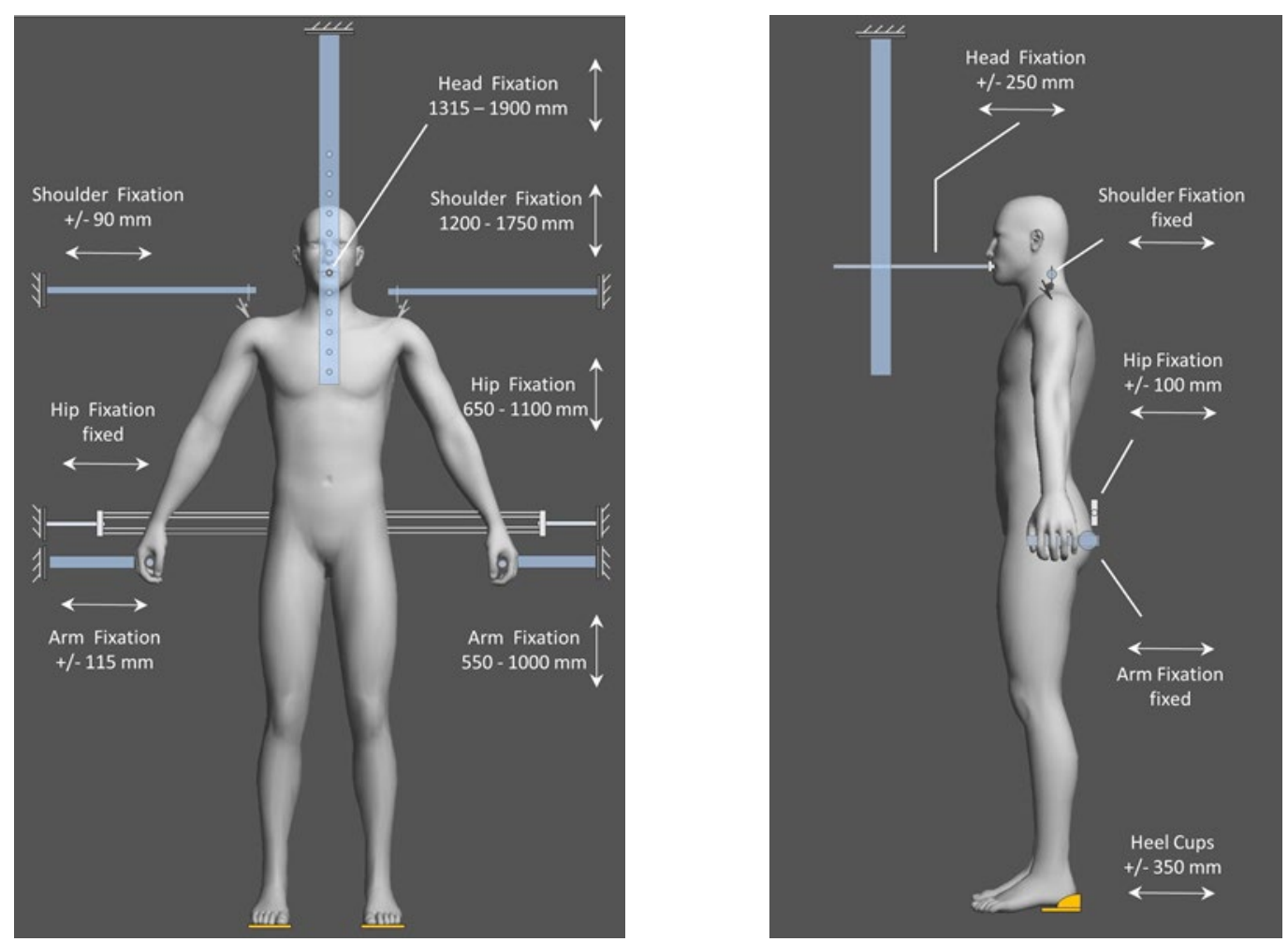

Figure 3: Adjustability of the positioning aid for the standing posture

\subsubsection{Positioning aid for the seated posture}

The anthropometric study was also to include scans in the seated posture, which required the development of a positioning aid specifically for this purpose. Since the fixation elements for the standing posture did not affect data integrity, the same design principles and functional requirements were applied to the seated posture. For the study, the seated posture according to ISO 7250-1 [29] was selected, which delivers the key seated body dimensions and therefore has been used in numerous large-scale anthropometric surveys [30-32]. ISO 7250-1 stipulates for the seated posture that the upper and lower legs have to be at a $90^{\circ}$ angle and both forearms are bent $90^{\circ}$ with hands in a fist. Figure 4 shows the final design of the positioning aid for the seated posture meeting the ISO 7250 requirements. The fixation elements deviating from those for the standing posture are depicted in Table 2 and their design is described in more detail below.

Stool - A commercially available height-adjustable stool was mounted on a base plate with four guiding pins on its bottom, creating a robust and easily detachable connection with the scanner platform. For the seated posture according to ISO 7250-1, the upper and lower legs have to be at a $90^{\circ}$ angle. Therefore, the stool and the heel cups were placed at a distance from each other that fulfilled this requirement. 

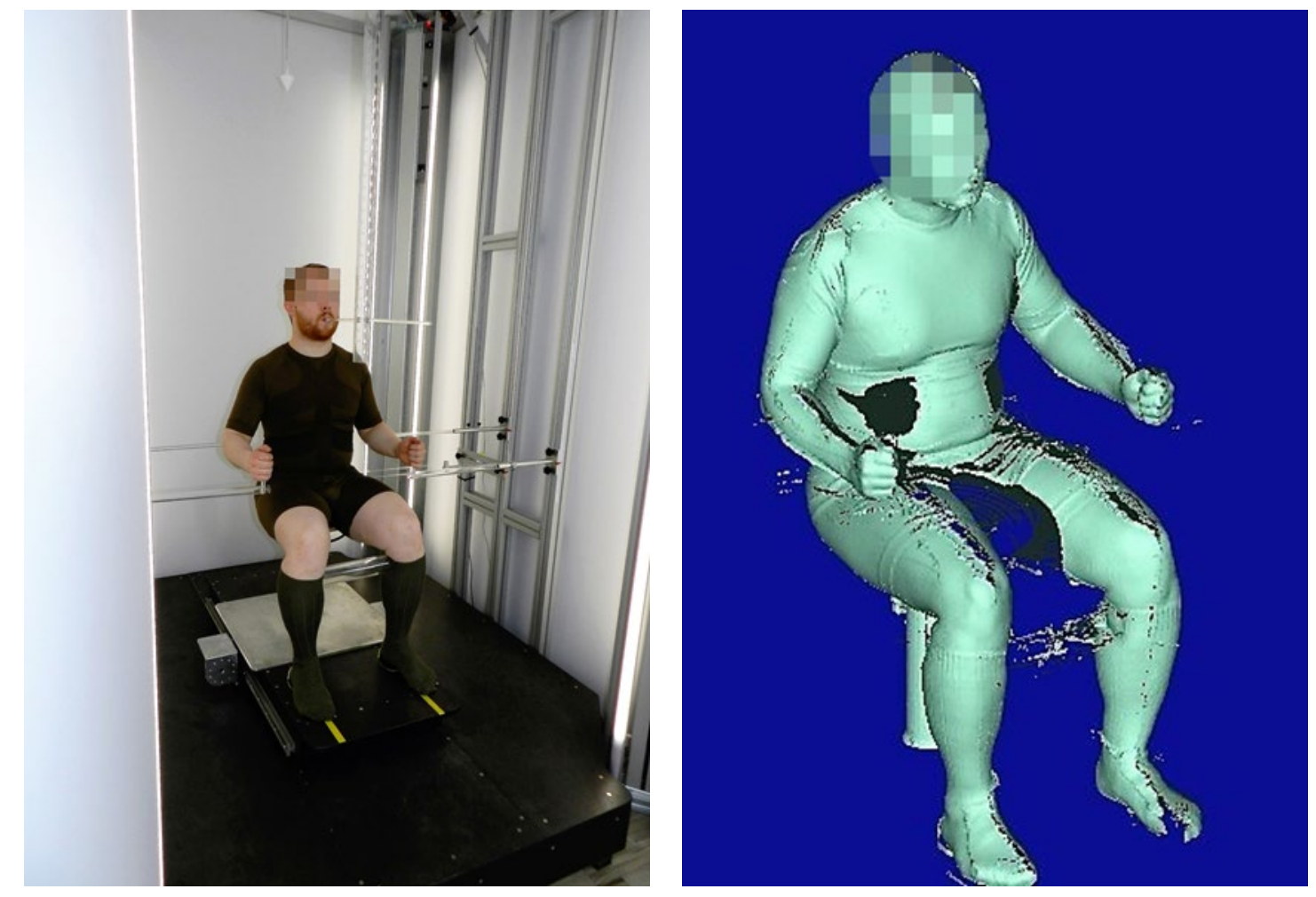

Figure 4: Positioning aid for the seated posture (left); Sample scan (right)

Head fixture - Due to the lower head position when sitting, the centre tube of the head fixation had to be extended compared to the standing posture. In order to ensure an adequate stiffness of the head fixation, the diameter and the thickness of the centre tube was increased.

Elbow fixture - To prevent variability of the upper arms in transverse direction a fixation element for the elbows was introduced. It was assumed that this new fixation element also stabilises the shoulder region of the participants and thus makes the shoulder fixation unnecessary. For the elbow fixation the design elements of the shoulder fixation for the standing posture, apart from the pivotal clip, were reused. The individual is expected to press the elbows against the end of the Plexiglas tube, which is adjusted to ensure that the right and left arm from elbow to fist are parallel and pointing to the front of the scanner.

Arm fixture - In the seated posture as per ISO 7250-1, both forearms are bent $90^{\circ}$ with hands in a fist. In contrast to the standing posture, this required the handles of the arm fixation to be turned into the upright position.

Knee tube - Like the elbows, the knees are susceptible to variability in the transverse direction. Therefore, a Plexiglass "knee tube" was introduced as an additional fixation element, the participants were expected to clamp between the lower legs. The upper edge of the tube was placed exactly at the height of the knee pit to ensure reproducibility.

Again, a visibility test was conducted to find out whether the design efforts led to the desired result. Table 2 gives an overview of the test results. 
Table 2: Evaluation of the fixation elements of the positioning aid for the seated posture in terms of visibility

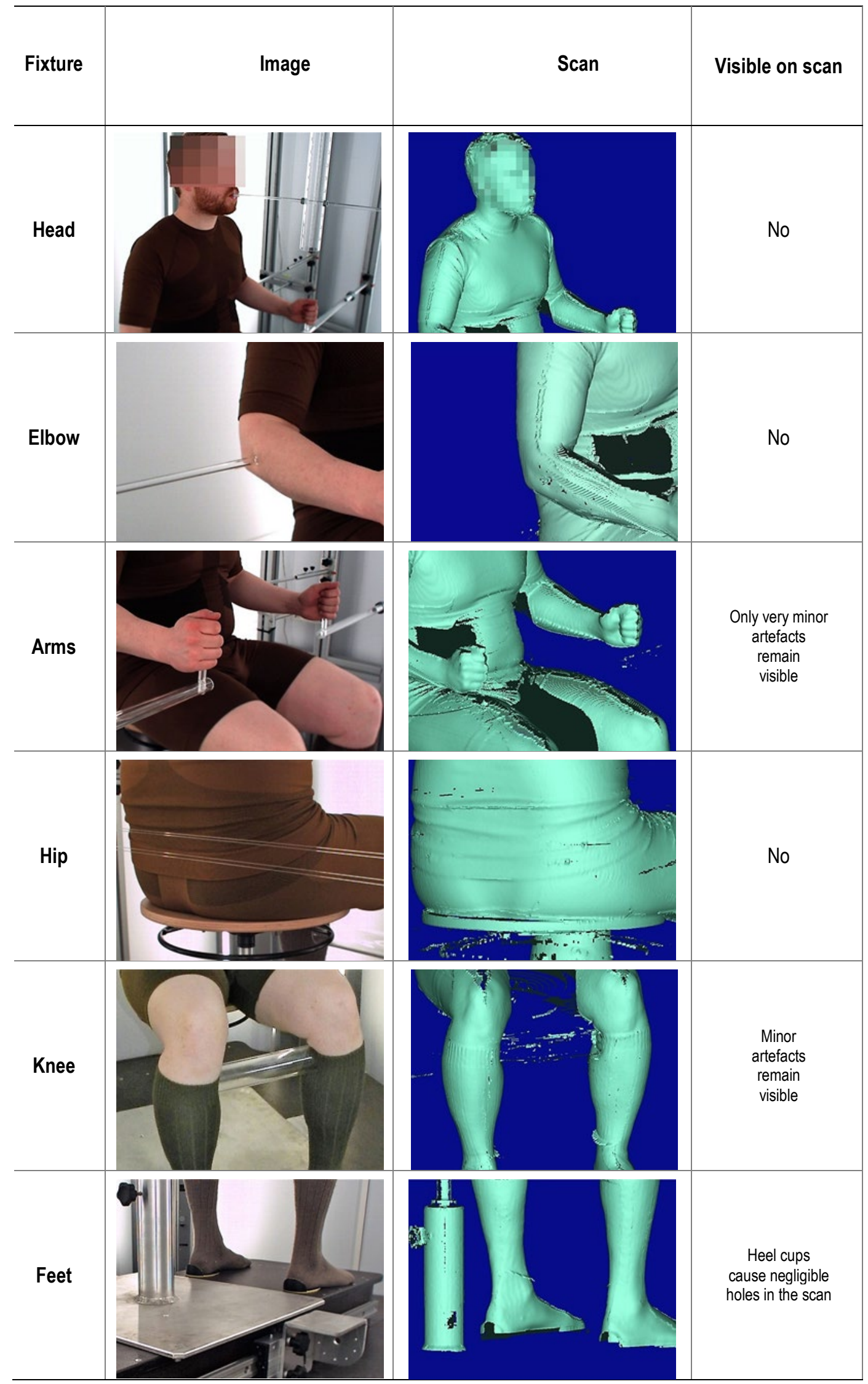


The adjustment ranges of the positioning aid for the seated posture are depicted in Figure 5.
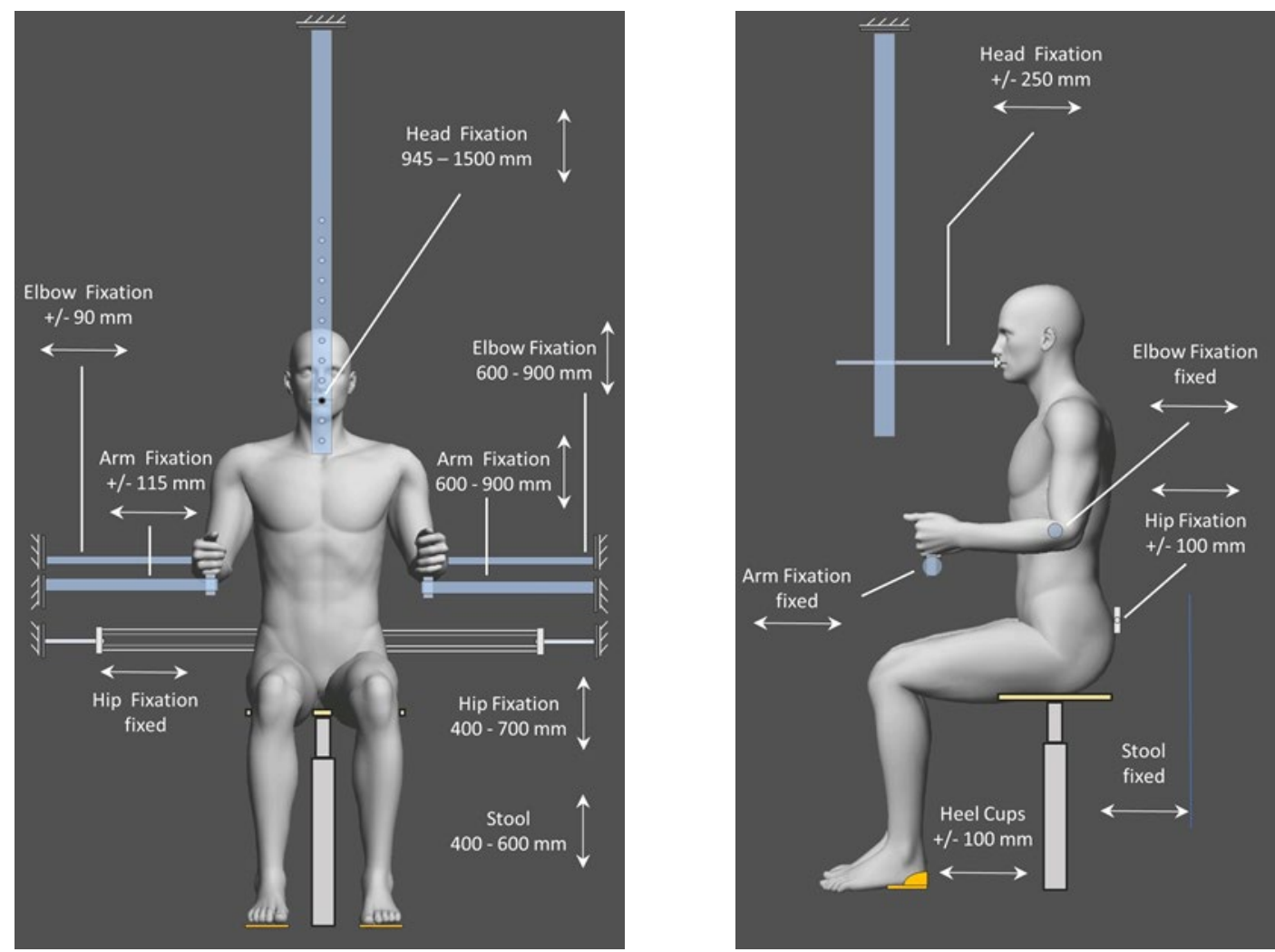

Figure 5: Adjustability of the positioning aid for the seated posture

\subsection{Methodology for evaluating the effectiveness of the positioning aid}

\subsubsection{Design of the experiment}

The human variability between repeated scans taken of the same participant in different clothing configurations has a decisive impact on the accuracy with which the offset between the clothing configurations can be determined. Previous research [19] showed that a positioning aid can effectively reduce the human variability and presented a methodology for quantifying its efficacy. This methodology also formed the starting point for the evaluation of the positioning aids developed for the high-resolution scanner. Consequently, the study included three semi-nude scans with and three without positioning aid in both the standing and the seated postures specifically to determine the efficacy with which the positioning aids reduce human variability.

On a voluntary basis 37 male participants from the German Army were recruited to participate in the study. The sample ranged in stature from 168.1 to $190.0 \mathrm{~cm}(M=179.6 \mathrm{~cm}, S D=5.9 \mathrm{~cm})$ and in weight from 63.1 to $146.8 \mathrm{~kg}(M=86.8 \mathrm{~kg}, S D=16.4 \mathrm{~kg})$. The tests were subject to the ethics regulations of Loughborough University, which included informed consent of the participants. The efficacy test took 40 minutes per participant, including the time required to adjust the fixation elements of the positioning aids to the physical dimensions. The four experimental conditions the 
same 37 participants were exposed to, i.e., with and without positioning aid in the seated and standing posture, differ fundamentally. This and the fact that the participants were requested after each of the three repeated scans to relax and reposition themselves avoided training or memory effects.

ISO 20685 [33] stipulates that "for all postures, quiet respiration (normal breathing) should be adopted". This is in line with the findings of the study conducted by Mckinnon and Istook [34], who scanned subjects holding their breath at different inhalation levels and when breathing normally. They found that humans are unable to reliably replicate a certain respiration level and that continuing breathing normally during the scanning process leads to the highest reproducibility. That is why the participants were requested to continue breathing normally throughout the scanning process.

\subsubsection{Reference Test}

A reference test was performed with a rigid mannequin resembling a human individual as reference object since it entirely eliminates the effects of human variability, i.e. respiration, body sway and poor replication of the posture. If suitably fixed to the floor, the deviations between multiple scans of the mannequin can be solely ascribed to the precision of the hardware and software of the scanning system. This technical variability is system inherent and constitutes the minimum variability that can be achieved with the experimental set-up and therefore is utilised as reference to the efficacy of the positioning aid. Due to the limited adjustability of the mannequin' $s$ extremities, no reference test could be performed in the seated posture. Limiting the reference test to the standard standing posture was considered acceptable since the technical variability is independent of the body posture (Figure 6). 


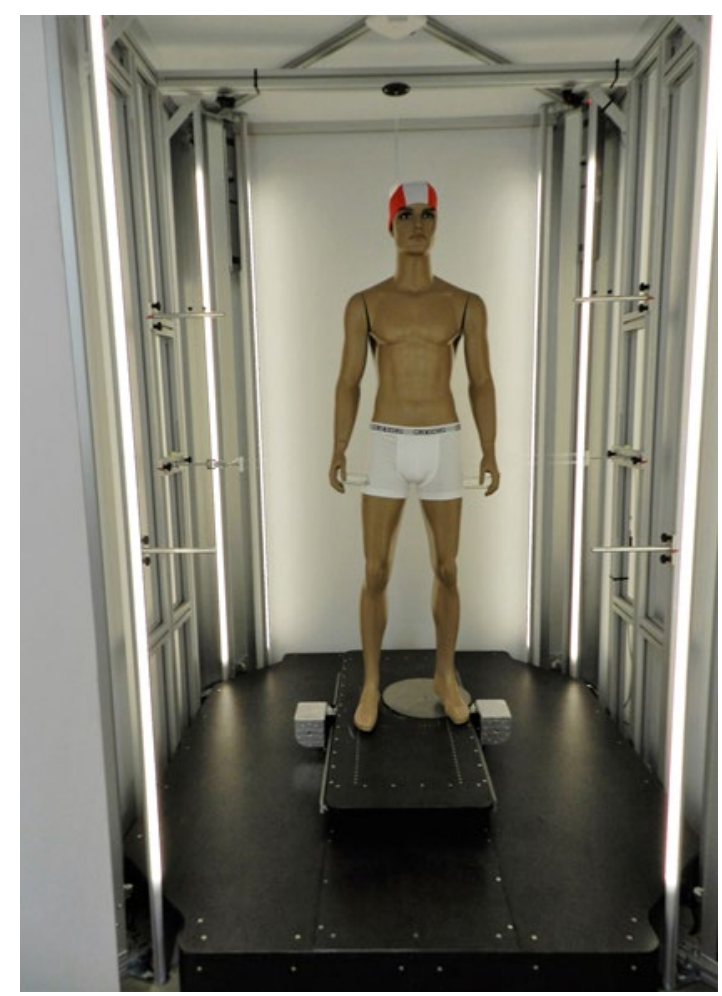

Figure 6: Rigid mannequin used for the reference tests

\subsubsection{Rating the variability by gauging sections}

The Anthroscan software package (HUMAN SOLUTIONS, Kaiserslautern, Germany), which forms an integral part of the scanning system, was used to automatically extract the landmarks shown in Figure 7 from the first of three scans both with and without positioning aid. Subsequently, scan two and three were superimposed and horizontal sections taken through seven of the previously extracted anatomical landmarks in the standing posture and six in the seated posture (Figure 7).
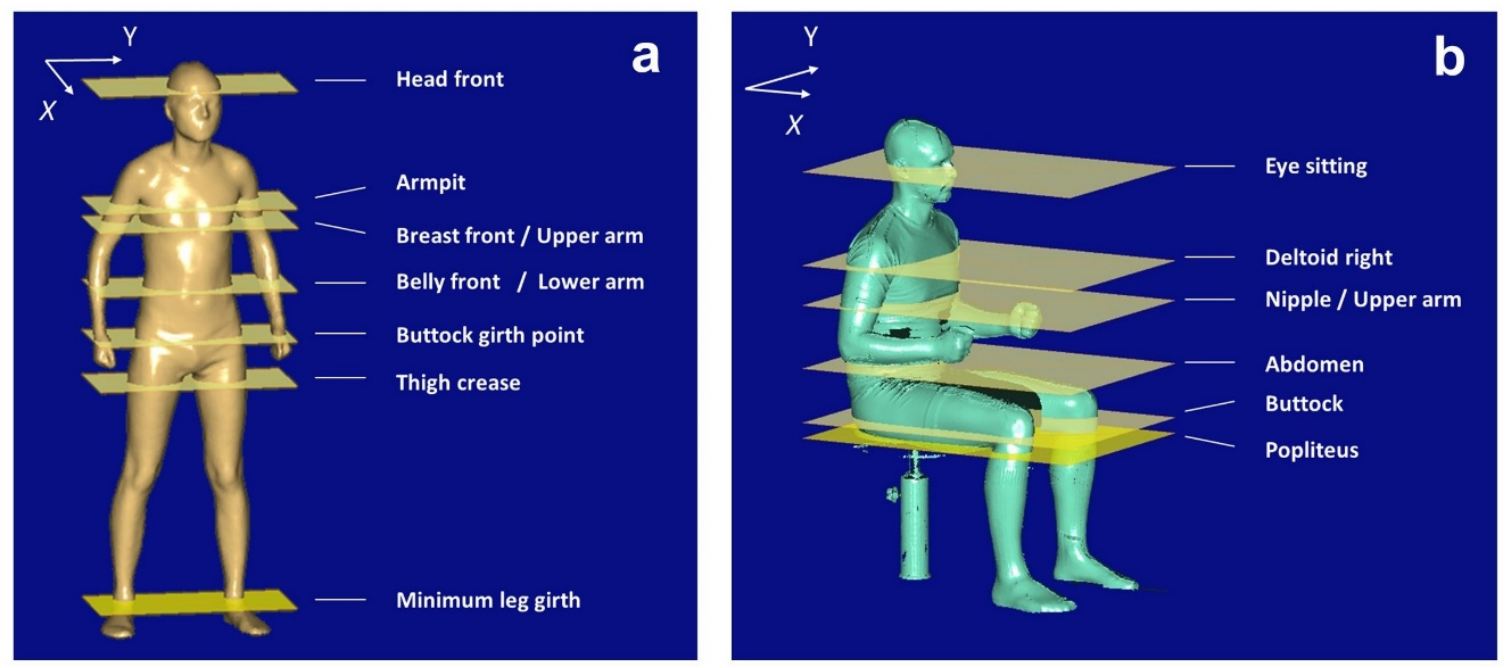

Figure 7: Anatomical landmarks through which sections were taken to evaluate the effectiveness of the positioning aids (a) for the standing posture and (b) the seated posture 
Contrary to earlier research [19], the software package PolyWorks (InnovMetric) was used instead of Anthroscan for taking and gauging horizontal sections. Compared to Anthroscan, PolyWorks has the advantage that different colours can be assigned to the sections of the individual scans (Figure 8b). In addition, PolyWorks allows the distances between the sections to be measured exactly along the sagittal (x) and transverse (y) axes (Figure 8b). In contrast to PolyWorks, Anthroscan only allows point-to-point measurements to be performed that can deviate from these axes (Figure 8a).
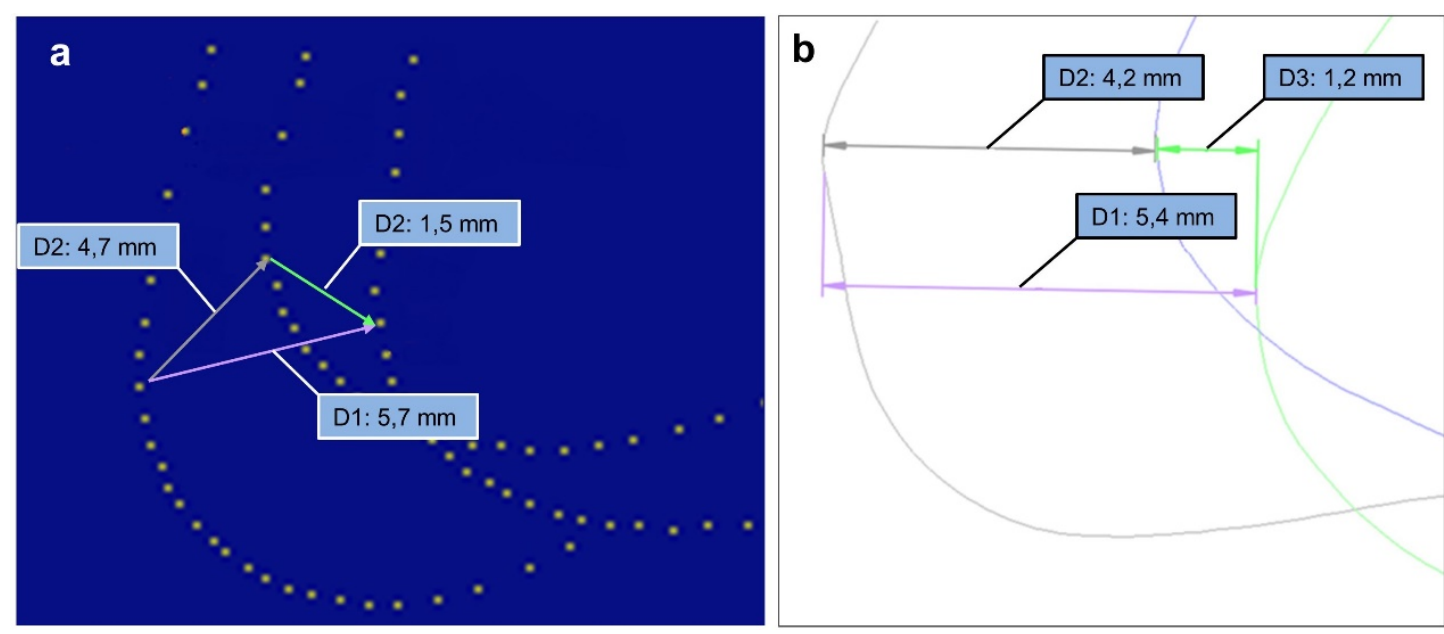

Figure 8: (a) Point to point measurements with Anthroscan; (b) measurements in exact x-direction and ydirection with PolyWorks

Due to these advantages, PolyWorks was utilised to measure the sagittal distances ( $\mathrm{x}$ ) and transverse distances $(\mathrm{y})$ between each section of the three superimposed scans in the two experimental conditions. Figure 9 illustrates this approach for the section through the head front landmark. 


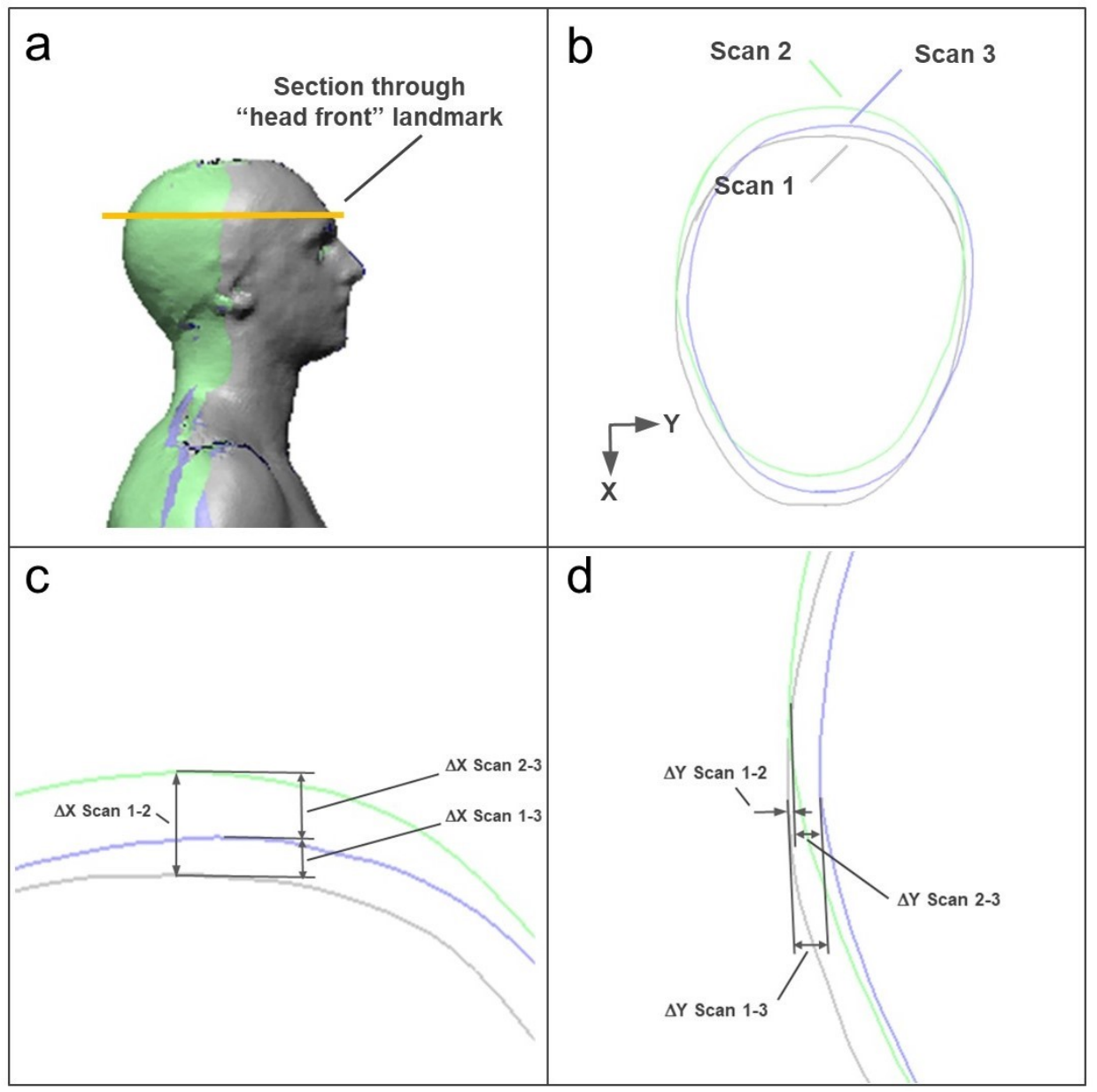

Figure 9: (a) Position at which the "head front" section is taken; (b) Superimposed "head front" sections; (c) Measurements in the sagittal direction; (d) Measurements in the transverse direction

\subsubsection{Mean absolute variability (MAV) as measure of variability}

In previous work, the authors evaluated the variability between repeated scans by determining the maximum distance between the contours of the two scans that represented the extremes of the three superimposed scans. The maximum distances always represented the worst case. However, this was unavoidable due to the fact that the Anthroscan software used for this purpose displayed all scans in the same colour and the superimposed contours could not always be clearly assigned to individual scans. As shown in the previous section, these limitations have been overcome by the use of PolyWorks, which allowed the distances between individual scans to be determined. This made it possible to calculate the mean value of the distances separately for both directions and both experimental conditions, thus introducing a more representative measure of the variability between repeated scans.

This approach was applied to the scans of each of the 37 participants in order to calculate the mean absolute variability $(M A V)$ across the sample for each of the selected body locations depicted in Figure 7. The $M A V$ index as a measure of the overall variability, i.e. human and technical variability, is defined as 


$$
M A V L_{S}=\frac{1}{n} \sum_{i=1}^{n}\left(\frac{\sum_{\mathrm{j}=1}^{\mathrm{r}} \sum_{k=1}^{r}\left|d_{i j k}\right|}{\frac{r !}{2(r-2) !}}\right), j>k
$$

Here, $M A V L_{S}$ denotes the mean absolute variability at body location $L$ for the entire sample $S$ with the sample size $n$ across the $r$ repeated scans ascertained from the $i^{\text {th }}$ participant. The variable $d_{i j k}$ represents the distance measured between the $j^{t h}$ scan and the $k^{\text {th }}$ scan. Defining that $j$ is greater than $k$ ensures that only the relevant measurements between $r$ superimposed scans are considered for calculating the mean absolute difference ( $r=3$ and $n=37$ in this study).

To quantify the technical variability, the same sections as those used for the human participant scans were reused on the scans of the mannequin and the mean variability between the superimposed scans measured.

\subsubsection{Statistical method}

A paired sample t-test was performed to test the sample differences for significance separately for the two positioning aids. To this end the MAV ixy $\Sigma L$ was calculated with and without positioning aid and used to determine the sample difference. The MAV ixy $\Sigma L$ denotes the mean absolute variability of the $i$ th participant across all body locations and both directions, i.e. the sagittal $(x)$ and transverse plane $(y)$. The null hypothesis was that there is no difference between the mean variability without positioning aid and the mean variability when using the positioning aid. The significance level $\boldsymbol{\alpha}$ was set at 0.05 . In addition, the effect size (Cohen $\boldsymbol{d}$ ) between the data obtained with and without positioning aid was evaluated based on Cohen's guidelines [35].

\section{Results and Discussion}

\subsection{Efficacy of the positioning aid for the standing posture}

The mean absolute variability across the sample $M A V L_{S}$ was calculated with and without positioning aid to evaluate its efficacy. The comparison of the variability with positioning aid $\left(M A V L_{S}\right.$ with $)$ and without $\left(M A V L_{S} / o\right)$ reveals the extent to which the positioning aid reduced the deviations between the three superimposed scans for the nine selected body locations. The calculations were conducted for the sagittal $\left(M A V L x_{S}\right)$ and transverse $\left(M A V L y_{S}\right)$ directions to identify possible directional dependencies. The reduction of variability by the positioning aid is a measure of its efficacy and is given in percent. In Table 3 the results are presented in the form of bar graphs to visualise the reduction in variability. 
Table 3: Variability of the sample in sagittal (MAVLxs) and transverse plane (MAVLys) with and w/o positioning aid for the standing posture

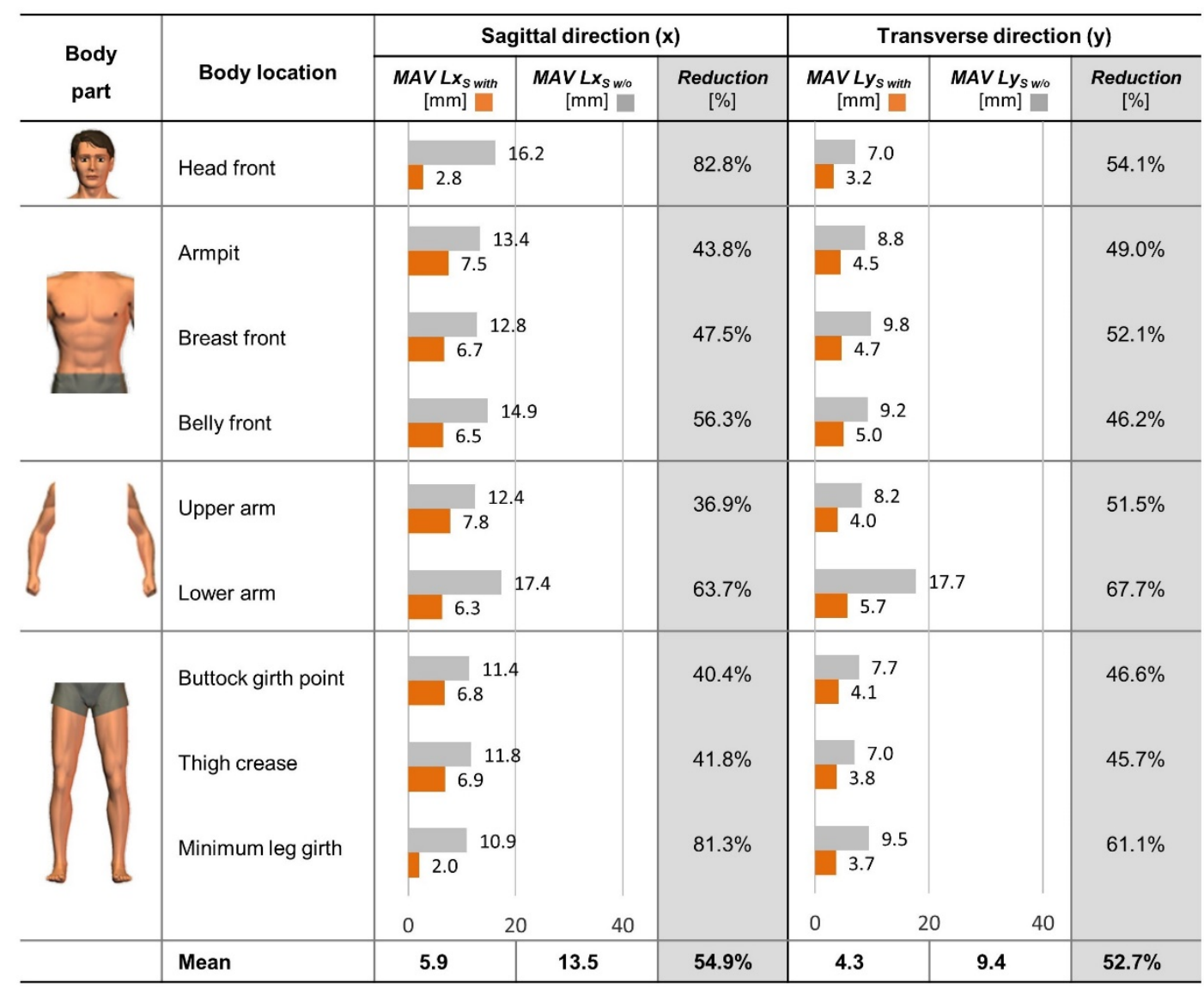

From Table 3 it is apparent that the variability with positioning aid does not exceed $7.8 \mathrm{~mm}$ in the $\mathrm{x}$-direction (upper arm) and $5.7 \mathrm{~mm}$ in the y-direction (lower arm). The lowest variability was measured in both the sagittal and transverse planes for the body locations head front $(2.8 \mathrm{~mm}$; $3.2 \mathrm{~mm}$ ) and $\mathrm{min}$ leg girth $(2.0 \mathrm{~mm} ; 3.7 \mathrm{~mm})$. The mean $M A V L_{S}$ with across all body locations amounts to $5.9 \mathrm{~mm}$ in the sagittal direction and $4.3 \mathrm{~mm}$ in the transverse direction. Utilising the positioning aid more than halved the overall variability in either direction $(54.9 \%(\mathrm{x}) ; 52.7 \%(\mathrm{y}))$. The highest reductions were observed in the sagittal direction at the body locations head front $(82.8 \%)$ and $\min$ leg girth $(81.3 \%)$. In the transverse direction, the greatest reductions were achieved for the lower arm (67.7\%) and min leg girth (61.1\%).

From the paired t-test results it can be inferred that utilising the positioning aid led to a significant reduction in variability $(\boldsymbol{t}(36)=7.0, \boldsymbol{p}<0.001$, one-tailed $)$. The mean decrease in variability was $5.7 \mathrm{~mm}$ with a $95 \%$ Confidence Interval (CI) ranging from $4.0 \mathrm{~mm}$ to $7.4 \mathrm{~mm}$. The Cohen $\boldsymbol{d}$ value (1.2) indicates a large effect size.

\subsection{Effectiveness of the positioning aid for the seated posture}

As for the standing posture, the variability with $\left(M A V L_{S}\right.$ with $)$ and without positioning aid $\left(M A V L_{S} w\right)$ was compared to determine the extent to which the positioning aid for the seated 
posture reduced the variability at the seven selected body locations. Again, the variability was measured in the sagittal $\left(M A V L x_{S}\right)$ and transverse $\left(M A V L y_{S}\right)$ directions (Table 4).

Table 4: Variability of the sample in sagittal $\left(M A V L x_{S}\right)$ and transverse (MAVLys) plane with and w/o positioning aid for the seated posture

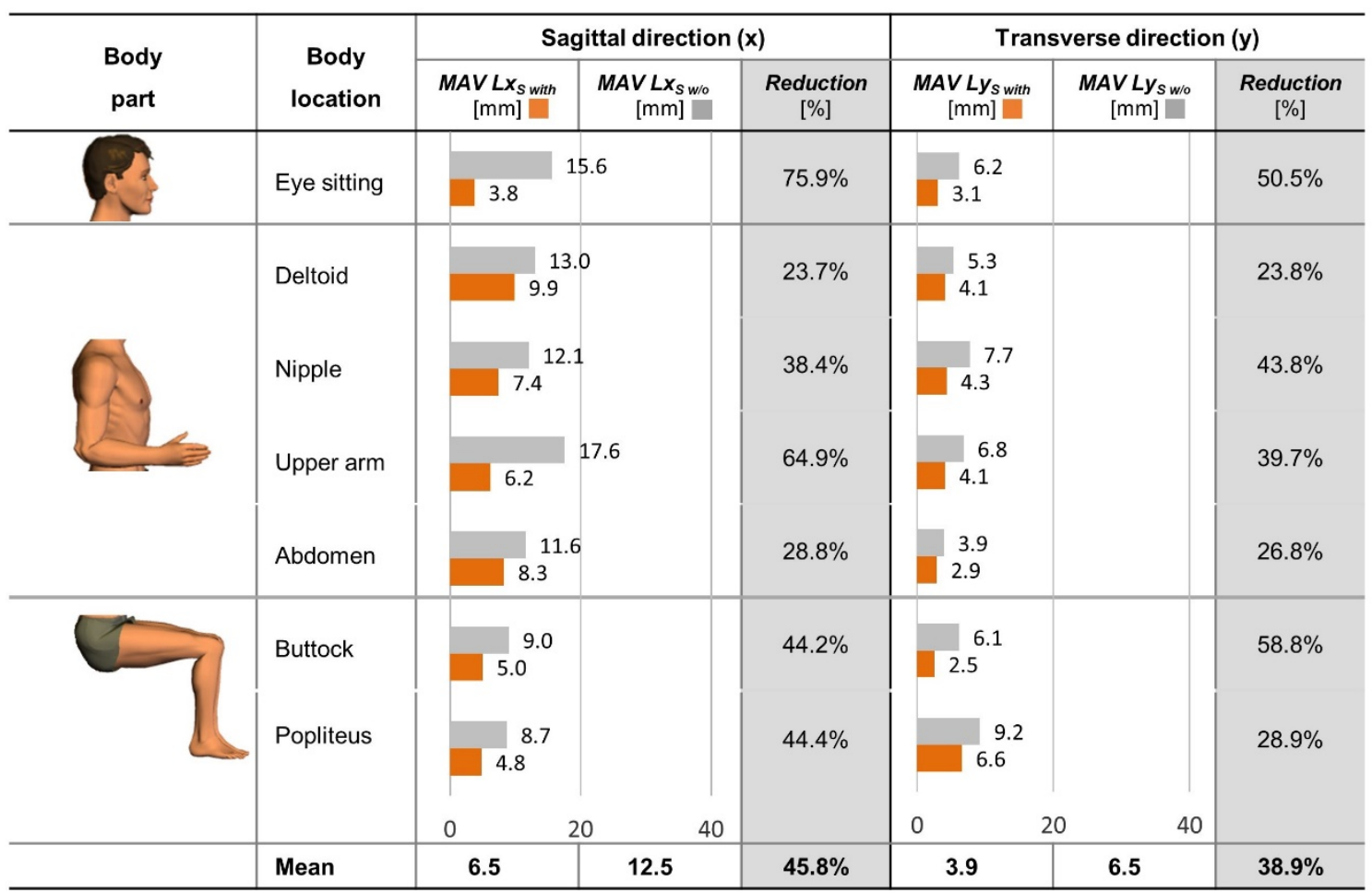

As can be seen from Table 4, the variability with positioning aid does not exceed $9.9 \mathrm{~mm}$ in the $\mathrm{x}$ direction (deltoid) and $6.6 \mathrm{~mm}$ in the y-direction (popliteus). The lowest variability was observed in the sagittal plane at the body location eye sitting $(3.8 \mathrm{~mm})$ and in the transverse plane at the buttock $(2.5 \mathrm{~mm})$. The mean $M A V L_{S}$ with across all body locations amounts to $6.5 \mathrm{~mm}$ in the sagittal direction and $3.9 \mathrm{~mm}$ in the transverse direction. The positioning aid reduced the overall variability by $45.8 \%$ in the sagittal direction and $38.9 \%$ in the transverse direction. The highest reductions were observed in the sagittal plane at the body locations eye sitting (75.9\%) and upper arm (64.9\%). In the transverse plane, the greatest reductions were achieved for the body locations buttock (58.8\%) and eye sitting (50.5\%).

The results of the paired t-test suggest that utilising the positioning aid for the seated posture significantly reduced the variability $(\boldsymbol{t}(36)=5.8, \boldsymbol{p}<0.001$, one-tailed). The mean decrease in variability was $3.8 \mathrm{~mm}$, with a $95 \% \mathrm{CI}$ ranging from $2.5 \mathrm{~mm}$ to $5.1 \mathrm{~mm}$. The effect size according to Cohen's standards was large (Cohen $\boldsymbol{d}=1.0)$.

\subsection{Contribution of the technical variability to the overall variability}

The mean absolute variability presented in Tables 3 and 4 encompasses both the technical variability caused by the hardware and software of the scanning system as well as the human 
variability of the scanned individual, i.e., body sway, variations in respiration level and postural variability. To quantify the extent to which the technical variability contributes to the overall mean absolute variability the reference test outlined in Section 2.2.2. was conducted. Scanning a rigid mannequin eliminated the human variability and permitted the system inherent technical variability of the scanning system to be determined. Since the reference test with the mannequin was only conducted in the standing posture, the evaluation was limited to this posture. To permit a succinct comparison, the mean of the sagittal (MAVLx) and transverse (MAVLy) variability was calculated as a direction-independent measure of variability $(M A V L x y)$. Table 5 presents a bar chart of the $M A V L y_{S}$ wo, MAV Lxys with and MAV Lxy Ref at the nine body locations relevant for the standing posture. Furthermore, the contribution of the technical variability MAV Lxy Ref to the residual variability with positioning aid MAV Lxys with constituting the $100 \%$-reference is provided.

Table 5: Contribution of the technical variability to the mean absolute variability with and w/o positioning aid for the standing posture

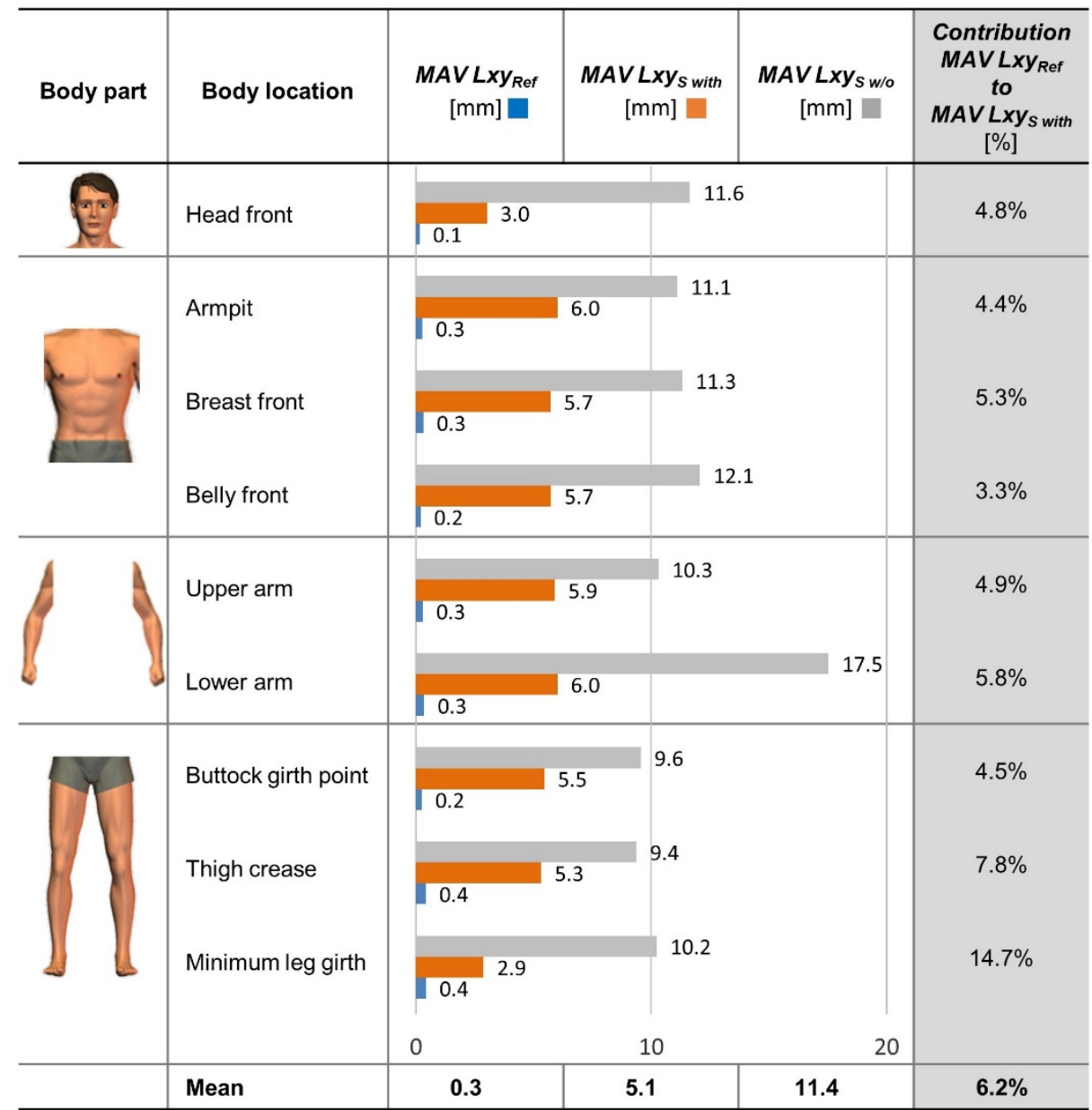

The table shows that the system-inherent technical variability does not contribute more than $0.4 \mathrm{~mm}$ at any of the body locations and stays well below the measurement accuracy specified by the scanner manufacturer $(<1 \mathrm{~mm})$. With $0.1 \mathrm{~mm}$ the contribution of the technical variability to the 
overall variability is particularly low at the body location head front. On average the technical variability contributed $6.2 \%$ to the residual variability with positioning aid.

\subsection{Discussion}

The evaluation based on gauging sections showed that the positioning aid for the standing posture effectively reduced the mean absolute variability between multiple scans for the nine selected body locations in sagittal (54.9\%) and transverse (52.7\%) directions. The lowest residual variability was observed for head and feet $(<3.8 \mathrm{~mm})$. At the remaining seven body locations the residual variability only varied by less than $1.5 \mathrm{~mm}$ in sagittal $(6.3 \mathrm{~mm}-7.8 \mathrm{~mm})$ and $1.2 \mathrm{~mm}$ in transverse plane $(3.8 \mathrm{~mm}-5.0 \mathrm{~mm})$.

While the mean residual variability achieved with the positioning aids for the standing and seated posture do not differ significantly $(0.6 \mathrm{~mm}(\mathrm{x}) ; 0.4 \mathrm{~mm}(\mathrm{y}))$, the residual variability values observed in the seated posture are less balanced. The highest result occurred at the deltoid in the sagittal plane $(9.9 \mathrm{~mm}(\mathrm{x}))$. This is a strong indication that the elbow fixation did not stabilise the shoulder region to the same extent as the shoulder fixation of the positioning aid for the standing posture. However, the elbow fixation is indispensable to avoid variability of the lower arm in the transverse plane. Using, in addition, the shoulder fixation designed for the standing posture would increase the burden on the participants and limit the freedom of movement of the experimenter to adjust the fixation elements. Against this background, the slightly higher variability in the shoulder area is considered an acceptable trade-off for a lower burden on participants and experimenters.

The evaluation of the technical variability showed that, on average, it contributed only $6.2 \%$ to the residual variability with positioning aid. The contribution reported in earlier research for a VITUS $^{\text {smart }}$ LC laser scanner and a NX16 structured light scanner is about four times higher (23.1\%) and seven times higher respectively (40.4\%) [19]. This suggests that the high-resolution scanner used in the study constitutes a significant improvement in precision.

The mean variability observed without positioning aid in the VITUS ${ }^{\text {bodyscan }}\left(M A V\right.$ Lxy $\left._{S w / o}\right)$ is with $11.4 \mathrm{~mm}$ (Table 5) significantly lower than those obtained in earlier research [28] with a VITUS $^{\text {smart }}$ LC $(16.3 \mathrm{~mm})$ laser scanner and a NX16 $(16.9 \mathrm{~mm})$ structured light scanner . Due to light sensitivity the scans with the NX16 and VITUS ${ }^{\text {smart }}$ LC had to be taken at low illumination levels. In contrast, the VITUS ${ }^{\text {bodyscan }}$ allows scans to be taken in normal light conditions. As reported by Rugelj et al. [36], body sway is more intense in darkness than at normal illumination levels. This offers a plausible explanation for the differences in variability, which is why even higher reductions in variability can be expected when using the newly developed positioning aids in combination with light-sensitive scanning systems. 
Generally speaking, the mouthpiece and the heel cups showed to be particularly effective at positioning the head and feet respectively. Also, the support elements selected for the remainder of body locations effectively reduced the variability to a balanced level for both positioning aids. Further increasing the fixation of the trunk, arms and thighs in order to bring them closer to the level of variability of the head and feet poses a challenge. On the one hand, increasing the fixation level constitutes an additional physical burden on the human subjects. On the other hand, additional fixation elements present further hindrances for the experimenter and extend the time needed to adjust the positioning aid (2-3 min per fixation element).

As far as the methodology for determining the efficacy of the positioning aids are concerned the introduction of the $\boldsymbol{M A V}$ provides advantages over those used in earlier research:

- Compared to the $M A D$ index, which rates the variability between repeated scans by calculating the absolute difference between body dimensions extracted from these scans, the measurement chain is shorter and does not repeatedly include the error prone automatic landmark detection. Furthermore, the $M A V$ provides information on the extent to which the variability manifests in sagittal and transverse planes.

- Due to the limitations of the software, in previous studies the mean maximum variability between repeated scans was used to characterise the variability. However, this represents the most unfavourable case. In contrast, the $M A V$ provides an average and thus more representative and meaningful information about the accuracy that can be expected when superimposing scans taken of an individual restrained by a specific positioning aid.

It needs to be noted, however, that the $M A V$ as a measure of efficacy for the positioning aid generates results in the range $<10 \mathrm{~mm}$. Particularly against the background of using positioning aids to determine the offset between semi-nude and clothed configurations, in which significantly higher variations occur due to bulk and different ways of wearing multi-layered clothing and equipment, the question arises, whether "searching for the mm" makes sense. Compared to the $M A V$, the mean maximum variability ( $M M V)$ across the sample determined from the two scans of an individual representing the extremes at the different body locations, is approximately twice as high. The maximum variability (Max.V) observed for individual participants ranged between 20 and $30 \mathrm{~mm}$ with positioning aid and even over $100 \mathrm{~mm}$ without [28]. This demonstrates that the $M M V$ and the Max.V are the more appropriate measures to describe the magnitude of the reduction in postural variability that can be achieved by specific positioning aids. They impressively demonstrate that the postural deviations without positioning aids would prevent a meaningful measurement of the offset between superimposed scans taken in different clothing configurations. The accuracy of the measurement chain for determining the offset between garment configurations is composed of the accuracy with which 
the positioning aids allow the replication of the posture and the accuracy of the software used for the actual measurement of the 1D, 2D or even 3D space claim differences. As mentioned above, unlike the $M M V$ or Max.V, the $M A V$ does not represent an extreme, but indicates the average accuracy with which postures can be replicated when using a specific positioning aid. It is an analogy to the $M A D$, which has been established as a benchmark for the reproducibility of repeated scan-derived measurements [24-26]. This makes the $M A V$ ideally suited as measure of efficacy for positioning aids in reducing postural variability, which at the same time quantifies the accuracy in the overall measurement chain.

A limitation of the $M A V$ is that it constitutes a simple point-to-point index albeit in the exact $\mathrm{x}$ $\mathrm{y}$ planes. Besides the shifting in $\mathrm{x}$ - or $\mathrm{y}$-direction, a rotation of the superimposed scans relative to each other was observed. The introduction of a measure of rotation would allow the extent to which the positioning aid prevents the individual body parts from being twisted to be determined. This would provide a further criterion besides the $M A V$ for evaluating the efficiency of positioning aids.

\section{Conclusions and further work}

In this paper the development of positioning aids for the standing and seated posture for the use in combination with a high-resolution scanner is described that do not compromise data integrity. Utilising specialised software, the mean absolute variability (MAV) index has been introduced to determine the efficacy of the positioning aids and thus overcome limitations of previously used measures of efficacy. The introduction of an additional rotational measure, which describes to what extent the superimposed sections are rotated relative to each other, could serve as complementary criterion for evaluating the efficacy of positioning aids.

The results presented in Tables 3 and 4 revealed that in the standing posture the mean absolute variability of $7.9 \mathrm{~mm}$ at the upper arm in sagittal plane and in the seated posture of $9.9 \mathrm{~mm}$ at the deltoid in sagittal plane constitute the worst cases when using the positioning aids. It therefor can be concluded that both positioning aids reduced the human variability to an extent that permit scans to be superimposed with an accuracy of $<8 \mathrm{~mm}$ in the standing and $<10 \mathrm{~mm}$ in the seated posture. This way the precondition was created to collect 3D data of the same individual semi-nude and clothed with postural variations reduced to an extent that allows space-claim differences to be measured with the appropriate level of accuracy. Hence, the logical progression of the research described in this paper would be to explore options to best determine the linear, circumferential and volumetric space claim differences between scans taken of the same individual with positioning aids in diverse clothing configurations (Figure 10). 

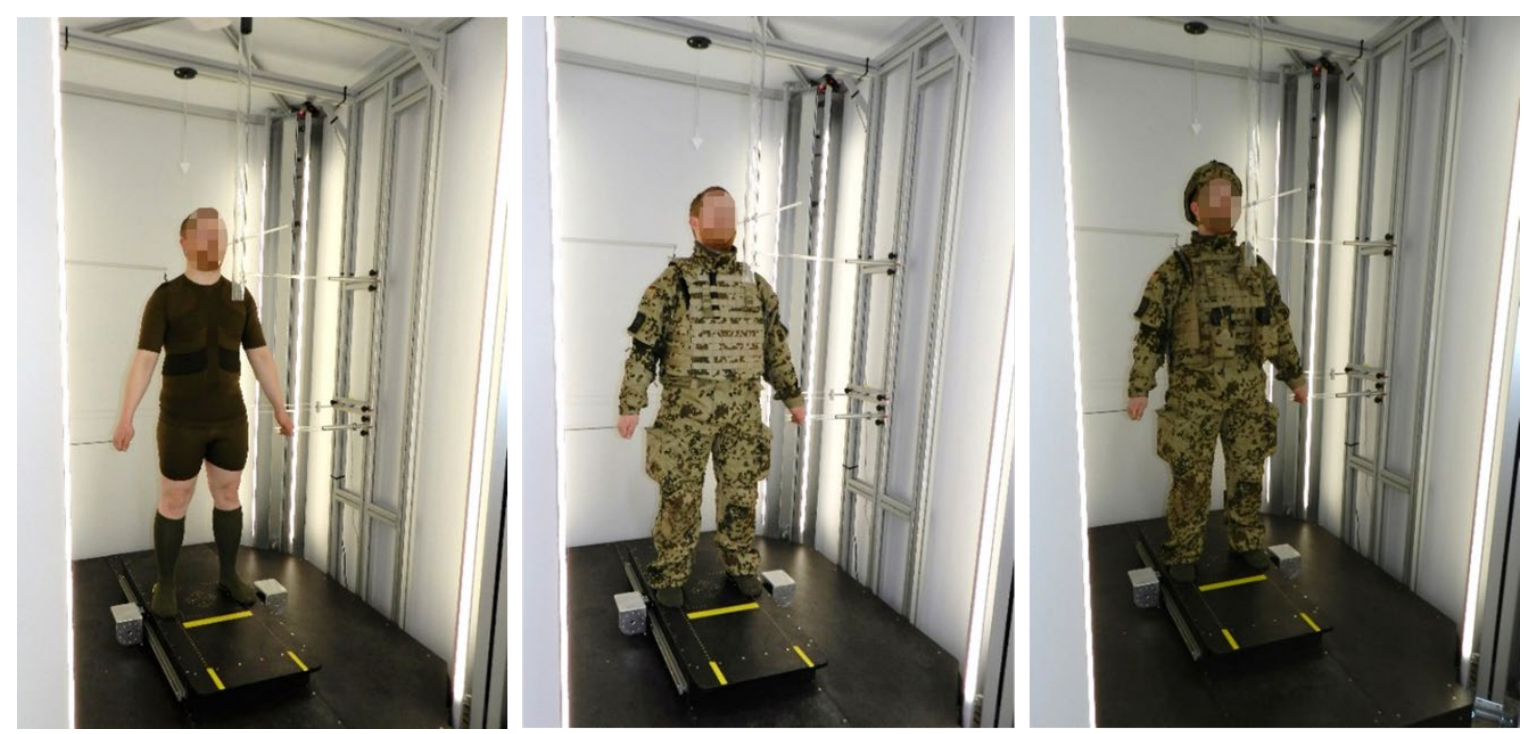

Figure 10: Participant scanned in divers clothing configurations: (a) semi-nude; (b) with combat jacket, combat trousers and ballistic vest; (c) fully encumbered

\section{Funding}

The unconditional support of the Bundeswehr Technical Centre for Land-Based Vehicle Systems, Engineer and General Field Equipment (WTD 41) and the Mechanized Infantry of the Bundeswehr is gratefully acknowledged. 


\section{References}

[1] VITRONIC GmbH, Technical data sheet VITRONIC bodyscan laser scanner, VITUS Bodyscan, High-Precision, Fast Color 3D Scans. (2020). https://www.vitronic.de/download/pdfs/3d-bodyscanner.html.

[2] H.A.M. Daanen, F.B. Ter Haar, 3D whole body scanners revisited, Displays. 34 (2013) 270275. https://doi.org/10.1016/j.displa.2013.08.011.

[3] A. Ballester, M. Valero, B. Nacher, A. Pierola, P. Piqueras, M. Sancho, G. Gargallo, J.C. Gonzales, S. Alemany, 3D Body Databases of the Spanish Population and its Application to the Apparel Industry, Proc. 6th Int. Conf. 3D Body Scanning Technol. Lugano, Switzerland, 27-28 Oct. 2015. (2015) 232-233. https://doi.org/10.15221/15.232.

[4] M. Almousa, The Development of a National Anthropometric Data Bank for Saudi Adults: The Initial Stage, Proc. 7th Int. Conf. 3D Body Scanning Technol. Lugano, Switzerland, 30 Nov.-1 Dec. 2016. (2016) 266-268. https://doi.org/10.15221/16.266.

[5] H.P. Lee, S. Garg, N. Chhua, F. Tey, Development of an Anthropometric Database Representing the Singapore Population, in: Proc. 6th Int. Conf. 3D Body Scanning Technol. Lugano, Switzerland, 27-28 Oct. 2015, 2015: pp. 234-241. https://doi.org/10.15221/15.234.

[6] R. Carrier, P. Meunier, Effects of protective equipment on anthropometric measurements and functional limitations, Rep. Def. Civ. Inst. Environ. Med. No.-CR-22, North York, Ontario, Canada. (1996).

[7] J.W. Kozey, C.J. Brooks, S.L. Dewey, R.C. Brown, K.A. Howard, D. Drover, S. MacKinnon, J. McCabe, Effects of human anthropometry and personal protective equipment on space requirements, Occup. Ergon. 8 (2009) 67-79. https://doi.org/10.3233/OER-2009-0160.

[8] M.L.H. Jones, P.S.E. Farrell, A. Keefe, Encumbered Anthropometry Protocol Development, in: Proc. Int. Ergon. Soc. Digit. Hum. Model. Symp., Ann Arbor, USA, 2013.

[9] S. Kamal, Relationship of Anthropometric Measurements Taken on Clothing to Those Taken on Body, Karachi Univ. J. Sci. 14 (1986) 35-39. https://www.researchgate.net/publication/278782853_Relationship_of_Anthropometric_M easurements_Taken_on_Clothing_to_Those_Taken_on_Body.

[10] A. Psikuta, J. Frackiewicz-Kaczmarek, E. Mert, M.-A. Bueno, R.M. Rossi, Validation of a novel 3D scanning method for determination of the air gap in clothing, Measurement. 67 
(2015) 61-70. https://doi.org/10.1016/j.measurement.2015.02.024.

[11] E. Mert, A. Psikuta, M. Arévalo, C. Charbonnier, C. Luible-Bär, M.A. Bueno, R.M. Rossi, A validation methodology and application of 3D garment simulation software to determine the distribution of air layers in garments during walking, Meas. J. Int. Meas. Confed. 117 (2018) 153-164. https://doi.org/10.1016/j.measurement.2017.11.042.

[12] B.D. Park, B.D. Corner, M. Kearney, M.P. Reed, Estimating human body characteristics under clothing using a statistical body shape model, Proc. 4th Int. Digit. Hum. Model. Conf. (2016) 1-6.

[13] J. Yang, J.S. Franco, F. Hétroy-Wheeler, S. Wuhrer, Estimation of human body shape in motion with wide clothing, Lect. Notes Comput. Sci. (Including Subser. Lect. Notes Artif. Intell. Lect. Notes Bioinformatics). 9908 LNCS (2016) 439-454. https://doi.org/10.1007/978-3-319-46493-0_27.

[14] C. Zhang, S. Pujades, M. Black, G. Pons-moll, Detailed, accurate, human shape estimation from clothed 3D scan sequences MPI for Intelligent Systems, T “, Cvpr. (2017) 4191-4200.

[15] A. Ballester, E. Parrilla, J. Uriel, A. Pierola, S. Alemany, B. Nacher, J. Gonzalez, J.C. Gonzalez, 3D-Based Resources Fostering the Analysis, Use, and Exploitation of Available Body Anthropometric Data, Proc. 5th Int. Conf. 3D Body Scanning Technol. Lugano, Switzerland, 21-22 Oct. 2014. (2014) 237-247. https://doi.org/10.15221/14.237.

[16] M. Colaianni, M. Zollhöfer, J. Süßmuth, B. Seider, G. Greiner, A Pose Invariant Statistical Shape Model for Human Bodies, in: 5th Int. Conf. 3D Body Scanning Technol., 2014: pp. $21-22$.

[17] Guitierrez, C. M., P.D. Gallagher, Pilot Study of Firefighter Three- Dimensional Anthropometry to Improve Seatbelt Safety (Report NIST 08-919), Gaithersburg, 2008.

[18] M.P. Reed, A Pilot Study of Three-Dimensional Child Anthropometry for Vehicle Safety Analysis, Proc. Hum. Factors Ergon. Soc. Annu. Meet. 56 (2012) 2326-2330. https://doi.org/10.1177/1071181312561489.

[19] F. Schwarz-Müller, R. Marshall, S. Summerskill, Development of a positioning aid to reduce postural variability and errors in 3D whole body scan measurements, Appl. Ergon. 68 (2018) 90-100. https://doi.org/10.1016/j.apergo.2017.11.001.

[20] S. Paquette, J.D. Brantley, B.D. Corner, P. Li, T. Oliver, Automated Extraction of Anthropometric Data from 3D Images, Proc. Hum. Factors Ergon. Soc. Annu. Meet. 44 
(2000) 727-730. https://doi.org/10.1177/154193120004403811.

[21] K. Robinette, H.A.M. Daanen, Precision of the CAESAR scan-extracted measurements., Appl. Ergon. 37 (2006) 259-65. https://doi.org/10.1016/j.apergo.2005.07.009.

[22] B. Bradtmiller, M.E. Gross, 3D Whole Body Scans: Measurement Extraction Software Validation, in: SAE Trans., 1999. https://doi.org/10.4271/1999-01-1892.

[23] L. Dekker, 3D Human Body Modelling from Range Data, University College London, 2000. https://discovery.ucl.ac.uk/id/eprint/1363354.

[24] J.-M. Lu, M.-J.J. Wang, The Evaluation of Scan-Derived Anthropometric Measurements, IEEE Trans. Instrum. Meas. $59 \quad$ (2010) 2048-2054. https://doi.org/10.1109/TIM.2009.2031847.

[25] M. Kouchi, M. Mochimaru, Errors in landmarking and the evaluation of the accuracy of traditional and 3D anthropometry., Appl. Ergon. 42 (2011) 518-27. https://doi.org/10.1016/j.apergo.2010.09.011.

[26] R.E. Sims, R. Marshall, D.E. Gyi, S.J. Summerskill, K. Case, Collection of anthropometry from older and physically impaired persons: Traditional methods versus TC2 3-D body scanner, Int. J. Ind. Ergon. 42 (2012) 65-72. https://doi.org/10.1016/j.ergon.2011.10.002.

[27] H. Han, Y. Nam, Automatic body landmark identification for various body figures, Int. J. Ind. Ergon. 41 (2011) 592-606. https://doi.org/10.1016/j.ergon.2011.07.002.

[28] F. Schwarz-Müller, Expedient Methodology for Capturing Anthropometric Data of Encumbered Personnel Utilising 3D Scanning Technology, Loughborough University, 2019.

https://repository.lboro.ac.uk/articles/Expedient_methodology_for_capturing_anthropomet ric_data_of_encumbered_personnel_utilising_3D_scanning_technology/12199084.

[29] BSI Standards Publication, BS EN ISO 7250 - 1: Basic human body measurements for technological design, Part 1: Body measurement definitions and landmarks, 2017.

[30] C.C. Gordon, T. Churchill, C.E. Clauser, J.T. Mcconville, I. Tebbetts, R.A. Walker, 1988 Anthropometric Survey of U . S . Army Personnel: Methods and Summary Statistics, Security. (1988) 640.

[31] S. Paquette, C. Gordon, B. Bradtmiller, Anthropometric survey (ANSUR ) II pilot study, 2009.

https://books.google.de/books?id=cb04LAY9yjgC\&dq=S.+Paquette, + C. + Gordon,,+ B. + Br 
adtmiller,+Anthropometric+survey+(ANSUR+)+II+pilot+study,+2009.\&lr=\&hl=de\&sour ce $=$ gbs_navlinks_s.

[32] A. Keefe, H. Angel, B. Mangan, Final Report - 2012 Canadian Forces Anthropometric Survey, $\quad$ DRDC-RDDC-2015-R186, $2015 . \quad$ https://cradpdf.drdcrddc.gc.ca/PDFS/unc328/p803174_A1b.pdf.

[33] BSI Standards Publication, BS EN ISO 20685-1: 3-D scanning methodologies for internationally compatible anthropometric databases, 2018.

[34] L. Mckinnon, C.L. Istook, Body scanning: The effects of subject respiration and foot positioning on the data integrity of scanned measurements, J. Fash. Mark. Manag. 6 (2002) 103-121. https://doi.org/10.1108/13612020210429458.

[35] J. Cohen, A Power Primer, Psychol. Bull. [PsycARTICLES]; 112 (1992) 155-159. https://doi.org/10.1038/141613a0.

[36] D. Rugelj, G. Gomišček, F. Sevšek, The influence of very low illumination on the postural sway of young and elderly adults, PLoS One. 9 (2014). https://doi.org/10.1371/journal.pone.0103903. 